\title{
A parallel complex divider architecture based on DCD iterations for computing complex division in MVDR beamformer
}

\author{
KIDAV Jayaraj $\mathrm{U}^{1,2, *}$, SIVA Mangai $\mathrm{N} \mathrm{M}^{1}$, and PERUMAL M Pillai \\ 1. Karunya Institute of Technology and Sciences, Coimbatore 641114, India; \\ 2. National Institute of Electronics and Information Technology, Calicut 673601, India
}

\begin{abstract}
This paper presents a hardware architecture using mixed pipeline and parallel processing for complex division based on dichotomous coordinate descent (DCD) iterations. The objective of the proposed work is to achieve low-latency and resource optimized complex divider architecture in adaptive weight computation stage of minimum variance distortionless response (MVDR) algorithm. In this work, computation of complex division is modeled as a $2 \times 2$ linear equation solution problem and the DCD algorithm allows linear systems of equations to be solved with high degree of computational efficiency. The operations in the existing DCD algorithm are suitably parallel pipelined and the performance is optimized to 2 clock cycles per iteration. To improve the degree of parallelism, a parallel column vector read architecture is devised. The proposed work is implemented on the field programmable gate array (FPGA) platform and the results are compared with state-of-art literature. It concludes that the proposed architecture is suitable for complex division in adaptive weight computation stage of MVDR beamformer. We demonstrate the performance of the proposed architecture for MVDR beamformer employed in medical ultrasound imaging applications.
\end{abstract}

Keywords: minimum variance distortionless response (MVDR) beamformer, adaptive weight, dichotomous coordinate descent (DCD) algorithm, medical ultrasound imaging.

DOI: $10.21629 /$ JSEE.2018.06.02

\section{Introduction}

Beamformer is a spatial filter and it is used to enhance the signal from a specific direction. In radar/medical ultrasound/sonar imaging applications, it is the most intensive processing block. Based on the imaging requirements, various beamforming algorithms are adopted. Adaptive beamformers provide significant improvements in directivity

\footnotetext{
Manuscript received June 17, 2017.

*Corresponding author.

This work was supported by Microelectronics Division of the Ministry of Electronics and Information Technology, Government of India, under SMDP-C2SD Project (9(1)/2014 -MDD).
}

and interference. Among adaptive beamformers for high resolution imaging, minimum variance distortionless response (MVDR) beamforming techniques are well renowned [1-4]. However, for high sampling rate applications like medical ultrasound imaging, embedded realtime realization of MVDR beamformer is an arduous task due to computational complexity of matrix inversion, complex division, etc [4]. In order to reduce the computational complexity, a QR decomposition (QRD) based sub array MVDR was proposed in [2]. Sub array MVDR degrades its performance in terms of main lobe width and peak side lobe level as compared to conventional MVDR. Fig. 1 depicts the general block diagram of MVDR beamformer. Here apodization weights are calculated based on certain adaptive algorithm. In MVDR beamformer, these weights are computed by (1). Here $\boldsymbol{S}(\boldsymbol{\Theta})$ is the steering vector and $\boldsymbol{R}^{-1} \boldsymbol{S}(\boldsymbol{\Theta})$ is the solution vector $\boldsymbol{h}$.

$$
\boldsymbol{W}(\boldsymbol{\theta})=\frac{\boldsymbol{R}^{-1} \boldsymbol{S}(\boldsymbol{\Theta})}{\boldsymbol{S}(\boldsymbol{\Theta})^{\mathrm{H}} \boldsymbol{R}^{-1} \boldsymbol{S}(\boldsymbol{\Theta})}
$$

In MVDR, the adaptive weight computation involves complex division (1), which is time critical and the most intensive operation as embedded implementation is concerned. In high sampling and data rate applications like radar/medical ultrasound, due to high computational complexity the system implementation is typically based on field programmable gate array (FPGA) or application specific integrated circuits (ASICs) rather than embedded processors. There are various methods available for computing complex division [5-8] in hardware. WANG et al. illustrated a high throughput complex divider implementation on FPGA based on coordinate rotation digital computer (CORDIC) [5]. Even though it provides high throughput, it consumes huge hardware resources; hence parallel implementation is not feasible. Edman et al. illustrated a multiplication- and division-free complex divider based on dichotomous coordinate descent (DCD) iterations 
[6]. Since it is multiplication- and division-free, it is most suitable for parallel implementation. Even though it is iterative, it allows linear systems of equations to be solved with high computational efficiency [9]. However, with the demonstrated area and throughput performance, adapting to hardware MVDR implementation is still challenging.

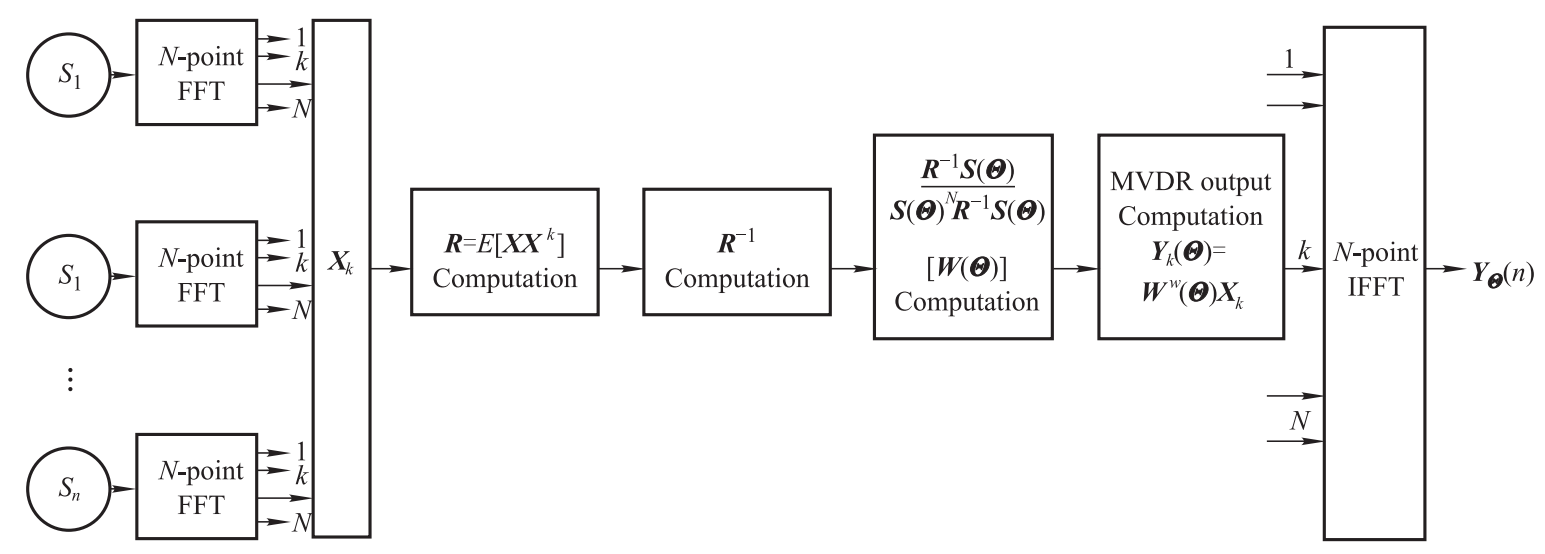

Fig. 1 General block diagram of MVDR beamformer

The subsequent sections discuss the DCD algorithm and DCD for complex division.

\subsection{DCD algorithm}

The DCD algorithm is an iterative method for solving linear systems of equations of the form $\boldsymbol{A x}=\boldsymbol{b}$, where $\boldsymbol{A}$ is an $N \times N$ matrix, $\boldsymbol{x}$ and $\boldsymbol{b}$ are $N \times 1$ column vectors. Here a sequence of better approximations $\boldsymbol{x}^{(k)}$ ( $k$ is the iteration index) will be produced, which hopefully converge $\boldsymbol{A x}=\boldsymbol{b}$ to an optimal solution. The idea behind coordinate descent (CD) is that the minimization of a multivariate function $f(\boldsymbol{x})$ can be achieved by minimizing it along one direction at a time, i.e., solving univariate optimization problems in a loop [8]. The CD iteration process is initiated by initializing $x^{0}=$ $\left(x_{1}^{0}, x_{2}^{0}, \ldots, x_{N}^{0}\right)$, then compute $\boldsymbol{x}^{k+1}$ iteratively from $\boldsymbol{x}^{k}$ by solving the single variable optimization problems $x_{i}^{k+1}=\arg \min _{\boldsymbol{b} \in \boldsymbol{R}} f\left(x_{1}^{k+1}, \ldots, x_{i-1}^{k+1}, b, x_{i+1}^{k}, \ldots, x_{N}^{k}\right)$ where $i$ varies from 1 to $N$. Thus the iteration starts for an initial $\boldsymbol{x}^{0}$ for a local minimum of $f(\boldsymbol{x})$ and gets the sequence $\boldsymbol{x}^{0}, \boldsymbol{x}^{1}, \boldsymbol{x}^{2}, \ldots$, etc. iteratively until an optimum solution $\boldsymbol{x}$ for $\boldsymbol{A} \boldsymbol{x}=\boldsymbol{b}$ satisfies.

The following depicts the algorithm in detail [10].

Initialize $\boldsymbol{\theta}=\boldsymbol{b}, \boldsymbol{x}=0, d=H, p=0$

for $m=1: M$

$$
\begin{aligned}
& d=d / 2 \\
& \text { do } \\
& \text { flag }=0 \\
& \text { for } j=0: N-1 \\
& \text { if }|\theta(j)|>d / 2 A(j, j) \\
& \text { flag }=1 ; p=p+1 \\
& x(j)=x(j)+\operatorname{sgn}(\theta(j)) d \\
& \theta=\theta-\operatorname{sgn}(\theta(j)) d(A(:, j))
\end{aligned}
$$

if $p>I_{\max }$ then stop

repeat while flag $=1$

Let $A(i, j), x(j)$ and $b(j)$ be the elements of matrix $\boldsymbol{A}$, vectors $\boldsymbol{x}$ and $\boldsymbol{b}$ respectively and $\boldsymbol{A}(:, j)$ be the $j$ th column of matrix $\boldsymbol{A}$. Before commencement of the iterative process, the solution vector $\boldsymbol{x}$ and an auxiliary vector $\boldsymbol{\Theta}$ are initialized to zero and $\boldsymbol{b}$ respectively. The initial set of iterations in the DCD algorithm determines the most significant bits for all elements of $\boldsymbol{x}$ using a step size parameter $d$ initialized to some value $H$, where $H$ is assigned a value, a power of two. The subsequent sets of iterations determine lower order bits until a suitable number of bits $M$ is obtained.

Suppose an iteration updates an element of solution vector $\boldsymbol{x}$, then the auxiliary vector $\Theta$ will also get updated. The processing complexity of the algorithm mainly depends on the successful updates of $\boldsymbol{x}$ and $\Theta$ vectors. To limit the successful iterations with an uncertain error in solution, a limit $I_{\max }$ can be predefined. If there is no such limit, then the accuracy of the solution is $2^{-M H}$. As described in pseudo code, the step size $d$ is a power of two. Hence only multiplication by a factor of two is needed and this can be implemented by bit shift operations without explicit multiplication or division operations. Hence the DCD algorithm is a hardware efficient algorithm for solving linear systems of equations.

\subsection{DCD algorithm for complex division}

The complex division problem $q=\frac{r}{d}$, where $q=q_{r}+\mathrm{j} q_{j}$, is the quotient, $r=r_{r}+\mathrm{j} r_{j}$ is the dividend and $d=d_{r}+\mathrm{j} d_{j}$ is the divisor, can be viewed as a problem of finding the solution of systems of linear equations of the form as shown 
in (2).

$$
\left(\begin{array}{cc}
d_{r} & -d_{j} \\
d_{j} & d_{r}
\end{array}\right)\left(\begin{array}{l}
q_{r} \\
q_{j}
\end{array}\right)=\left(\begin{array}{c}
r_{r} \\
r_{j}
\end{array}\right)
$$

Therefore, the solution for $q$ can be obtained by computing $q=d^{-1} r$; the same can be solved by the DCD algorithm. By referring the DCD algorithm explained in Section 1.1, the $2 \times 2 \mathrm{~d}$ matrix can be viewed as $\boldsymbol{A}$ matrix, $2 \times 1 q$ matrix as $\boldsymbol{x}$ and $2 \times 1 r$ matrix as $\boldsymbol{b}$ matrices.

The main contributions of this work are as follows:

(i) A parallel pipe lined complex vector division architecture, for adaptive weight computation in MVDR beamformer is proposed and demonstrated.

(ii) The existing FPGA implementation algorithm for complex cyclic valued DCD algorithm is optimized to perform two cycles per iteration and a parallel column vector read architecture is implemented to access correlation matrix memory.

(iii) The performance of the proposed architecture is compared with existing studies, and the architecture performance with respect to medical ultrasound imaging is demonstrated.

This paper is structured as follows:

Section 2 summarizes various complex division architectures implemented, justifying the advantages of DCD iterations for hardware implementation, necessity of complex division in broadband MVDR for medical ultrasound applications. Section 3 describes the proposed FPGA implementation algorithm for complex division. Section 4 briefs the very-large-scale integration (VLSI) architecture of the complex divider and architecture of various sub modules. Finally Section 5 presents the results and discussions and Section 6 presents the conclusions.

\section{Background}

Complex division is a fundamental operation to compute adaptive weight vector in MVDR beamformer. Embedded software-based implementation of complex division takes many processor cycles and satisfying high-speed real-time requirements of MVDR beamformer in medical ultrasound imaging is extremely difficult. Alternatively, FPGA-based designs are considered and performing the same is a complicated task in hardware [5]. When exploring VLSI architecture, high-throughput, low-latency and gate count (LUT and flip flops for FPGAs) are the major concerns on performance. In previous studies, several designs adopting various algorithms to address these performance issues have been presented.

In [5] a high throughput, fixed point complex divider is presented. In this implementation, the authors claim a $9 \times$ improvement in throughput as compared to the existing designs. Here four optimized CORDIC blocks are employed to compute complex division. The architecture is pipelined and the design is operating at a frequency of $113 \mathrm{MHz}$. After an initial latency of 19 cycles, further the architecture can perform division per clock cycle and hence a throughput of 113 millions of operations per second (MOPS) is claimed. As the architecture is based on CORDIC structures and CORDIC blocks consume more hardware resources, the design consumes much area. Edman et al. described complex division based on the Smith algorithm and realized based on real multipliers, adders and dividers [6]. The design could achieve a throughput of $28 \mathrm{MOPS}$ at $100 \mathrm{MHz}$ on Xilinx Virtex-II FPGA at the cost of 117 logic slices. The design is implemented in fixed point format (Q15) and the Smith algorithm does not guarantee accurate division results in fixed point implementation.

Efficient hardware realization of complex division is still under research and various iterative methods like Newton Raphson, and series expansion are adopted for hardware realization, but those realizations are costly in terms of hardware area and latency. Recent research has turned to use DCD algorithms for complex division, as complex division can be modeled as a linear equation, as shown in (2). Table 1 depicts comparison of various division algorithms for hardware realization.

Table 1 Performance comparison of various iterative algorithms for linear equation solution problem

\begin{tabular}{cccc}
\hline Algorithm & Iteration time & Latency (Cycles) & Area \\
\hline Newton Raphson & 2 serial multiplications & $\left(2\left[\log _{2} \frac{n}{i}\right]+1\right) t_{m u l}+1$ & 1 mul + table + control \\
Series expansion & 2 parallel multiplications & $\left(\left[\log _{2} \frac{n}{i}\right]+2\right) t_{m u l}+1$ & 1 mul + table + control \\
Accurate quotient approx. & 1 multiplication & $\left(\left[\frac{n}{i}\right]+1\right) t_{m u l}$ & 3 muls + table + control \\
Short reciprocal & 2 serial multiplications & $\left(2\left[\frac{n}{i}\right]\right) t_{m u l}+1$ & 1 short mul + table + control \\
Round/ pre scale & 1 multiplication & $\left(\left[\frac{n}{i}\right]+2\right) t_{m u l}+1$ & 1 mul + table + control \\
DCD algorithm & $\begin{array}{c}\text { Bit-shift operations, component } \\
\text { transpositions and negations }\end{array}$ & $(3 M b+n+2)$ & No mul and add \\
\hline
\end{tabular}


The DCD algorithm based complex division is implemented in [7]. Due to large number of iterations and consumption of many clock cycles per iteration, the throughput achieved is only 1.6 MOPS at a frequency of 100 MHz. The design is implemented on Virtex-II FPGA and 527 logic slices are utilized. Many complex division architectures are described in [5] but do not make much significance as MVDR beamformer implementation is concerned.

Many papers describe efficient complex matrix inversion for MVDR beamformer [2,11]. MVDR beamformer is a frequency domain realization and mostly beamformer output power can be computed in frequency domain itself, as shown in (3). Hence complex division needs not to be performed. However, while realizing broadband MVDR for medical ultrasound imaging applications [4], the output (4) should be in time domain, and hence adaptive weight and output need to be computed, and inverse fast Fourier transform needs to be performed. Therefore, there is a necessity to perform practically realizable high performance complex division.

$$
\begin{gathered}
P(\boldsymbol{\Theta})=\left[\boldsymbol{S}(\boldsymbol{\Theta})^{\mathrm{H}} \boldsymbol{R}^{-1} \boldsymbol{S}(\boldsymbol{\Theta})\right]^{-1} \\
Y(\boldsymbol{\Theta})=\boldsymbol{W}(\boldsymbol{\Theta})^{\mathrm{H}} \boldsymbol{X}
\end{gathered}
$$

Suppose the sampling frequency of a medical ultrasound system is $40 \mathrm{MHz}$, and the system can image at a maximum depth of $20 \mathrm{~cm}$, at a pulse repetition frequency of $4 \mathrm{kHz}$, and considering average velocity in soft tissues is $1540 \mathrm{~m} / \mathrm{s}$. Then the system needs to process approximately $10 \mathrm{~K}$ samples in $0.25 \mathrm{~ms}$. To satisfy the broadband criteria, $1 \mathrm{~K}$ point FFT based on array length [4] typically needs to be performed on each channel at an overlap of 12.5 percentage, i.e., 128 samples. Hence there would be approximately 13 FFT blocks which need to be processed. Due to the symmetry property of FFT, 512 bins need to be processed. For an $M$ channel system (Fig. 1) $13 \times 512$ bins need to be processed in $0.25 \mathrm{~ms}$. For each bin an $M \times 1$ weight vector needs to be generated. By employing parallel pipelined design for MVDR beamformer implementation on FPGA, the time to process 1 bin would be approximately $19.2 \mu$ s, i.e., the weight computation should be performed at a rate of $52 \mathrm{~K}$ operations per second.

The VLSI architecture implemented for broadband MVDR beamformer has various sub blocks like FFT processor, bin processor, adaptive weight computation logic, output computation logic and master controller. In addition to master controller, each sub blocks have their own controllers implemented to manage the data and control flow. The master controller manages the data and control flow among various sub blocks. If serial architecture for complex division is made to use, then the controller design would be complex and also the overhead in terms of number of clock cycles will be more. Therefore, even though the high throughput employing the architecture in [5] requires complex controller design, it also consumes huge FPGA resources. Also, serial implementation complex division needs to be updated in 27 MOPS to achieve the typical real-time performance requirements of the medical ultrasound system. Another advantage of the proposed implementation is that the same DCD-based architecture can be used to compute $\boldsymbol{R}^{-1} \boldsymbol{S}(\boldsymbol{\Theta})$. Therefore, the proposed complex division architecture can later be employed to develop adaptive weight computation core based on DCD iterations.

In Table $1, i$ is the number of bits of accuracy from an initial approximation, $t_{m u l}$ is the latency of the fused multiply/add unit in cycles, and $n$ is the number of iterations. As hardware implementation is concerned, adders and multipliers consume more chip area. Among these algorithms except DCD, all the other algorithms have adders and multipliers, so they consume large chip area and have high latency. It is possible to reduce the latency for certain computation, with a corresponding increase in system performance. Therefore, DCD is the best among these algorithms.

\section{The proposed FPGA algorithm}

FPGA implementation of the existing complex valued cyclic DCD algorithm is depicted in Table 2 [9]. Here the solution vector is $\boldsymbol{h}$ and it is evaluated by $\boldsymbol{h}=\boldsymbol{R}^{-1} \boldsymbol{\beta}$, where $\boldsymbol{R}$ is the complex correlation matrix and $\boldsymbol{\beta}$ is the steering vector.

\begin{tabular}{|c|c|c|}
\hline State & Operation & Cycle \\
\hline 0 & $\begin{array}{c}\text { Initialization: } \boldsymbol{h}=0, \boldsymbol{r}=\boldsymbol{\beta}, m=M \boldsymbol{b} \\
k=0, \Delta m=0, s=1, n=1\end{array}$ & \\
\hline 1 & $\begin{array}{c}\text { If } m=0, \text { algorithm stops else, } \\
m=m-1, \alpha=2^{\wedge} m, \Delta m=\Delta m+1\end{array}$ & 1 \\
\hline 2 & $\begin{array}{c}\text { If } s=1, \text { then } r t m p=R(r n) \\
\text { else, } \operatorname{real}(r(n))=\operatorname{img}(r n) c=R n \\
n / 2-|\operatorname{img}(r(n))| \times 2 \Delta m\end{array}$ & 1 \\
\hline 3 & $\begin{array}{c}\text { If } c<0, \text { then go to state } 4, \text { else, } \\
\text { go to state } 5\end{array}$ & 1 \\
\hline 4 & $\begin{array}{c}h n=h n+\operatorname{sign}(r t m p) s \alpha \\
r=r \times 2 \Delta m-\operatorname{sign}(r t m p) s \boldsymbol{R}(:, n) \\
\Delta m=0, k=k+1, \text { flag }=1 \\
\text { If } K=N u, \text { algorithm stops }\end{array}$ & $N$ \\
\hline 5 & $\begin{array}{c}\text { If } s=1, \text { then } s=j, \text { go to state } 2 \\
\text { else, } s=1, n=(n) \bmod (N / 2)+1 \\
\text { If } n=1 \text { and flag }=1, \text { then flag }=0, \\
\text { go to state } 2 \text {, else if } n=1 \text { and flag }=0, \\
\text { then go to state } 1 \text { else, go to state } 2\end{array}$ & 1 \\
\hline Total & $\leqslant 7 N N u / 2+3 N(M b-1)+$ Mbcycles & \\
\hline
\end{tabular}

Table 2 Existing complex cyclic valued DCD FPGA algorithm 
The matrix $\boldsymbol{R}$ and vectors $\boldsymbol{\beta}, \boldsymbol{r}$ and $\boldsymbol{h}$ are all complexvalued. The real and imaginary components of $r$ and $h$ are processed in parallel and each element is processed sequentially. A variable $s$ indicates which component is being processed; real $(s=1)$ or imaginary $(s=j)$.

This existing implementation has six states, and algorithm iterates from state 1 to state 5 . In state 4 , it takes $N$ (matrix size) clock cycles for $\boldsymbol{r}$ and $\boldsymbol{h}$ updation. Also the overall clock cycles to generate the solution are fewer than or equal to $7 N N u / 2+3 N(M b-1)+M b$ cycles, where $N u$ is the number of iterations and $M b$ is the number of solution vector bits.

An optimized implementation of the existing complex cyclic valued DCD algorithm is proposed and depicted in Table 3. Here the existing six state implementation is optimized to two states. The column vector and diagonal element can read simultaneously in one clock cycle from correlation matrix memory bank and hence only two clock cycles are required per iteration.

Therefore, the maximum number of clock cycles required is $2 N u$, where $N u$ is the number of iterations required to converge the algorithm. The modified algorithm is adopted to compute the adaptive weight in MVDR beamformer. The steps to compute adaptive weight are as follows:

The equation to generate adaptive weight is (1), where $\boldsymbol{W}=[1 \times N]$ matrix, $\boldsymbol{R}=[N \times N]$ matrix and $\boldsymbol{S}(\boldsymbol{\theta})=$ $[1 \times N]$ matrix. $N$ is the number of channels in an antenna array. Let the solution vector $\boldsymbol{h}=\boldsymbol{R}^{-1} \boldsymbol{S}(\boldsymbol{\theta})$ then equation to compute adaptive weight can be rewritten as

$$
\boldsymbol{W}=\frac{\boldsymbol{h}}{\boldsymbol{S}(\boldsymbol{\theta})^{\mathrm{H}} * \boldsymbol{h}}
$$

Now $\boldsymbol{h}$ is an $N \times 1$ matrix and $\boldsymbol{S}(\boldsymbol{\theta})^{\mathrm{H}} * \boldsymbol{h}$ is a complex value. Let $\boldsymbol{h}=\left[\left(a_{1}+\mathrm{j} b_{1}\right),\left(a_{2}+\mathrm{j} b_{2}\right),\left(a_{3}+\mathrm{j} b_{3}\right), \ldots,\left(a_{N}+\right.\right.$ $\left.\left.\mathrm{j} b_{N}\right)\right]$ and $\boldsymbol{S}(\boldsymbol{\theta})^{\mathrm{H}} * \boldsymbol{h}=s_{1}+\mathrm{j} k_{1}$, then

$$
\boldsymbol{w}=\left[\frac{a_{1}+\mathrm{j} b_{1}}{s_{1}+\mathrm{j} k_{1}}, \frac{a_{2}+\mathrm{j} b_{2}}{s_{1}+\mathrm{j} k_{1}}, \frac{a_{3}+\mathrm{j} b_{3}}{s_{1}+\mathrm{j} k_{1}}, \ldots, \frac{a_{N}+\mathrm{j} b_{N}}{s_{1}+\mathrm{j} k_{1}}\right]
$$

$\boldsymbol{w}$ can be computed by solving $N$ linear equations. Algorithm for adaptive weight computation using the modified cyclic valued DCD algorithm is depicted as follows.

Input: correlation matrix $\boldsymbol{R}$, steering vector $\boldsymbol{S}(\boldsymbol{\theta})$.

Initialize $H, M b, N u$.

Compute $R 1=\boldsymbol{R}^{\prime} * \boldsymbol{R}$.

Compute $q=\boldsymbol{R}^{\prime} \boldsymbol{S}(\boldsymbol{\theta})$.

Call pseudo code cyclic valued DCD algorithm to compute $\boldsymbol{h}=\boldsymbol{R}^{-1} \boldsymbol{S}(\boldsymbol{\theta})$.

Input: $R 1, q, H, M b, N u$
Table 3 Modified complex valued DCD FPGA algorithm

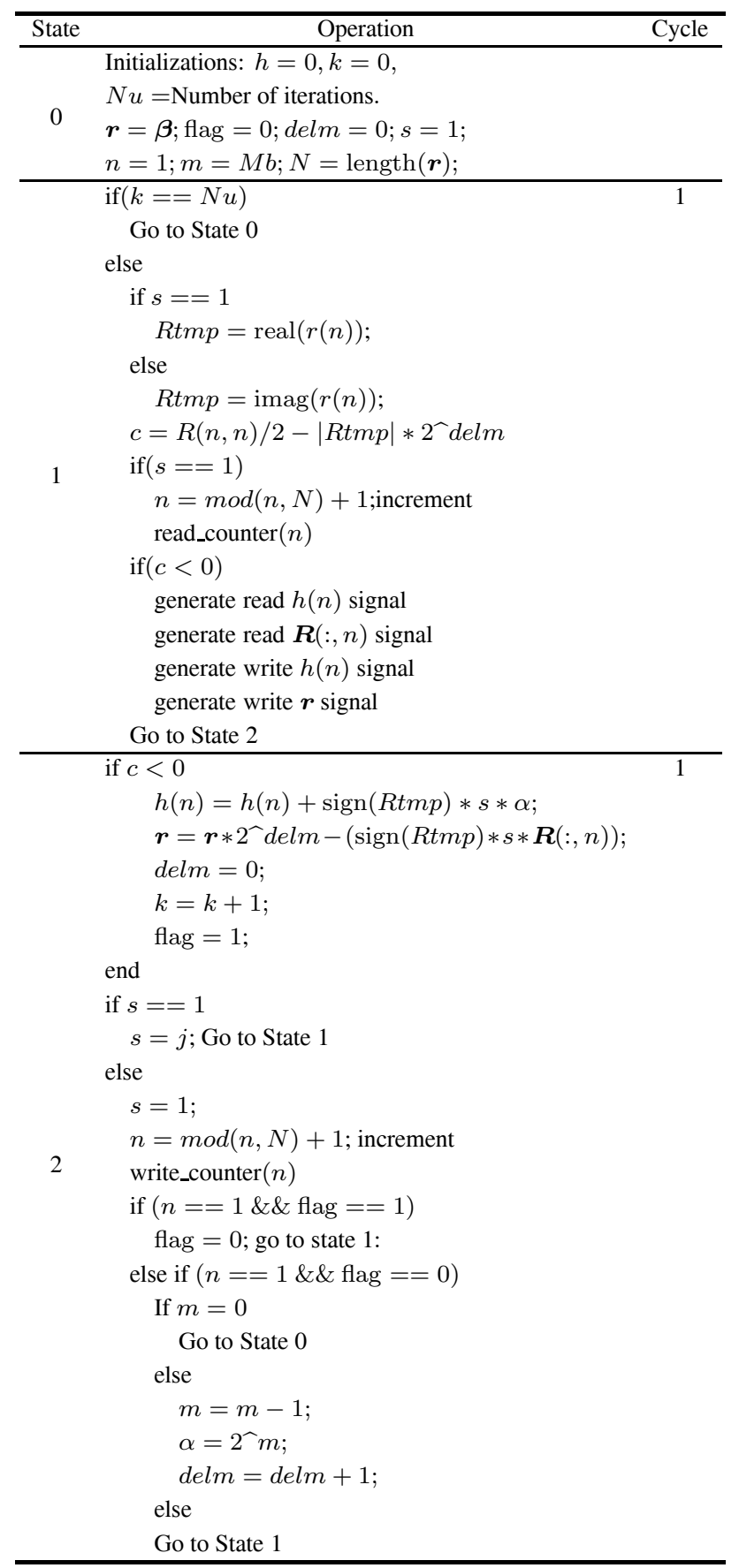

Output: $\boldsymbol{h}=\boldsymbol{R}^{-1} \boldsymbol{S}(\boldsymbol{\theta})$.

Compute $d=\boldsymbol{S}(\boldsymbol{\theta})^{\mathrm{H}} \boldsymbol{h}$.

Let $d r=\operatorname{real}(d)$ and $d j=\operatorname{imag}(d)$.

And $\boldsymbol{R}=[d r-d j d j d r]$

Compute new $R 1=\boldsymbol{R}^{\prime} * \boldsymbol{R}$;

Initialize $N=\operatorname{length}(h), \boldsymbol{w}=0$.

Repeat $i$ from 1 till $N$

$r r=\operatorname{real}(h(i))$;

$r j=\operatorname{imag}(h(i))$; 


$$
\begin{aligned}
& \boldsymbol{S}(\boldsymbol{\theta})=[r r r j] \\
& q=\boldsymbol{R}^{\prime} \boldsymbol{S}(\boldsymbol{\theta})
\end{aligned}
$$

Call pseudo code cyclic valued DCD algorithm to compute $h_{-} \boldsymbol{w}=\boldsymbol{R}^{-1} \boldsymbol{S}(\boldsymbol{\theta})$

Input: $R 1, q, H, M b, N u$

Output: $\boldsymbol{h} \_\boldsymbol{w}_{\boldsymbol{\prime}}=\boldsymbol{R}^{-1} \boldsymbol{S}(\boldsymbol{\theta})$.

Assign $w(i)=\operatorname{real}\left(\boldsymbol{h} \_\boldsymbol{w}\right)+\operatorname{imag}\left(\boldsymbol{h} \_\boldsymbol{w}\right) * \mathrm{i}$

The algorithm to solve the normal equation $\boldsymbol{R h}=\boldsymbol{S}(\boldsymbol{\theta})$ using the cyclic DCD algorithm is as follows:

Input: $R 1=\boldsymbol{R}^{\prime} * \boldsymbol{R}$ for $\boldsymbol{R}$ and $p=\boldsymbol{R}^{\prime} * \boldsymbol{S}(\boldsymbol{\theta})$ for $\boldsymbol{S}(\boldsymbol{\theta})$ in a system of $\boldsymbol{R h}=\boldsymbol{S}(\boldsymbol{\theta}) ; N u=$ Number of iterations.

Output: $\boldsymbol{R}^{-1} \boldsymbol{S}(\boldsymbol{\theta})$

Variables: $w, \alpha, k, r$, flag, delm, $s, n$

Initialize $w=0, m=M b, \alpha=H, k=0$, flag $=$ $0, s=1, n=1, N=$ length of steering vector, $r=p ; R=$ $R 1$;

$$
\begin{aligned}
& \text { while } m !=0 \\
& m=m-1 \\
& \alpha=2 \prec m \\
& \text { delm }=\text { delm }+1 \\
& \text { while }(k !=N u) \\
& \text { if } s==1 \\
& R t m p=\operatorname{real}(r(n)) \\
& \text { else } \\
& \text { Rtmp }=\operatorname{imag}(r(n)) \\
& c=R(n, n) / 2-((a b s(R t m p)) * 2 d e l m) \\
& \text { if } c<0 \\
& w(n)=w(n)+\operatorname{sign}(R t m p) * s * \alpha \\
& r=r * 2 \text { delm }-(\operatorname{sign}(\operatorname{Rtmp}) * s * \boldsymbol{R}(:, n)) \\
& \text { delm }=0 \\
& k=k+1 \\
& \text { flag }=1 \\
& \text { if } s==1 \\
& s=j \\
& \text { else } \\
& s=1 \\
& n=\bmod (n, N)+1 \\
& \text { if }(n==1 \& \& \text { flag }==1) \\
& \text { flag }=0 \\
& \text { else if }(n==1 \& \& \text { flag }==0)
\end{aligned}
$$

\section{Architecture design of the proposed complex divider}

VLSI architecture design and FPGA implementation are presented in this section. The proposed architecture is implemented on XILINX Kintex-7 XC320T FPGA and synthesized to $200 \mathrm{MHz}$ frequency. Fig. 2 depicts the architecture of the complex divider logic to compute adaptive weight in MVDR beamformer. The various units are steering vector register array, solution vector register array, complex vector multiplier and accumulator (MAC) logic, DCD divisor computation logic, DCD dividend computation logic, and DCD core and adaptive register array. The steering vector $\boldsymbol{S}(\boldsymbol{\Theta})$ and solution vector $\boldsymbol{h}$ are stored in steering vector register array and solution vector register array respectively. Both the steering vector and solution vector are complex and hence the register arrays contain real as well as imaginary registers.

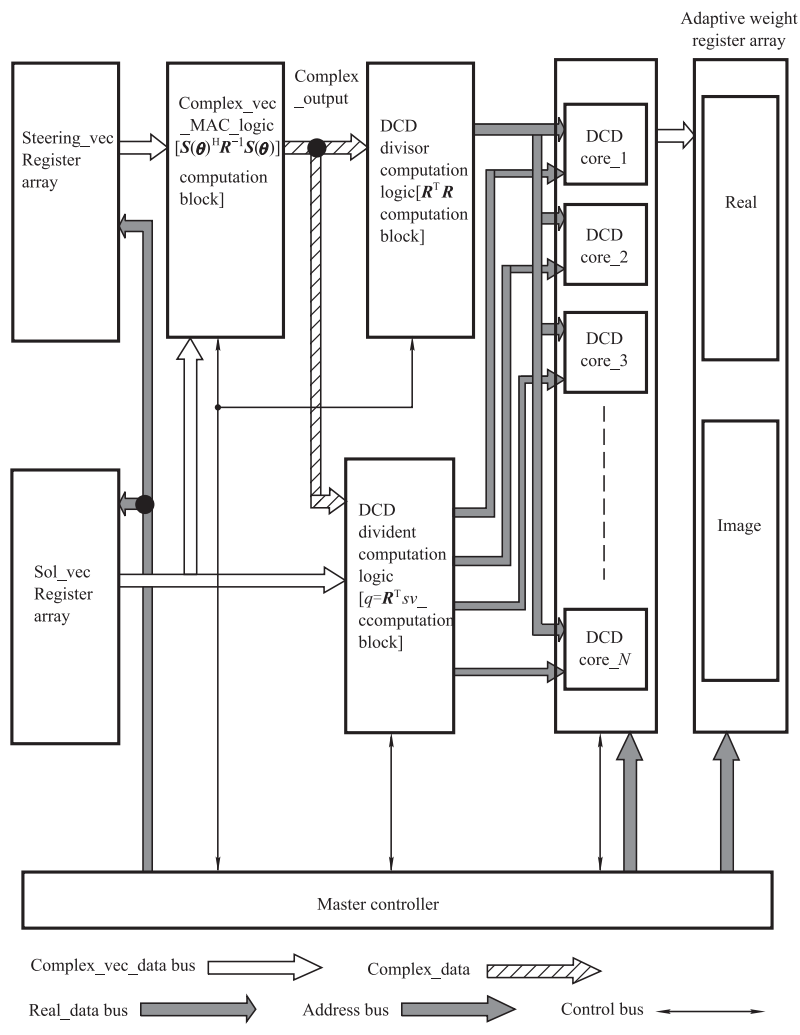

Fig. 2 Complex division logic for adaptive weight computation

MAC logic is multiplier and accumulation logic, and the complex vector MAC logic accepts input from steering vector register array and solution vector register array and computes $\boldsymbol{S}(\boldsymbol{\Theta})^{\mathrm{H}} \boldsymbol{R}^{-1} \boldsymbol{S}(\boldsymbol{\Theta})$. The output of MAC logic $(d r+\mathrm{i} d j)$ is fed to divisor computation and it computes $\boldsymbol{R}^{\mathrm{T}} \boldsymbol{R}$, where $\boldsymbol{R}$ is [ $\left.d r-d j ; d j d r\right]$. The inputs of dividend computation logic are from the output of MAC logic and solution vector register array and it computes $\boldsymbol{R}^{\mathrm{T}} \boldsymbol{S}(\boldsymbol{\Theta})$. The output of DCD divisor computation logic and DCD dividend computation logic are given to $32 \mathrm{DCD}$ cores and the output of DCD cores are written to adaptive weight register array.

The master controller is designed as a finite state machine and it generates the control signals to all units in the adaptive weight computation logic. The master controller generates address to steering vector register array, solution vector register array, DCD core and adaptive 
weight register array, and the required control signals to complex vector MAC logic $\left(\boldsymbol{S}(\boldsymbol{\theta})^{\mathrm{H}} \quad \boldsymbol{R}^{-1} \boldsymbol{S}(\boldsymbol{\Theta})\right)$ computation block, DCD dividend computation block $(q=$ $\boldsymbol{R}^{\mathrm{T}} s v_{-}$computation bloc), DCD divisor computation logic $\left(\boldsymbol{R}^{\mathrm{T}} \boldsymbol{R}\right.$ computation block) and 32 DCD core logic.

The sub module wise description of complex division logic for adaptive weight computation is described as follows.

\subsection{DCD core for complex division}

The DCD core (Fig. 3) contains three arithmetic (steering vector updater, solution vector updater and $C$ computation block), and memory (correlation matrix, solution vector and steering vector) units. The sequencing of data and control among memory units and arithmetic units are performed by a DCD controller, implemented as finite state machine.

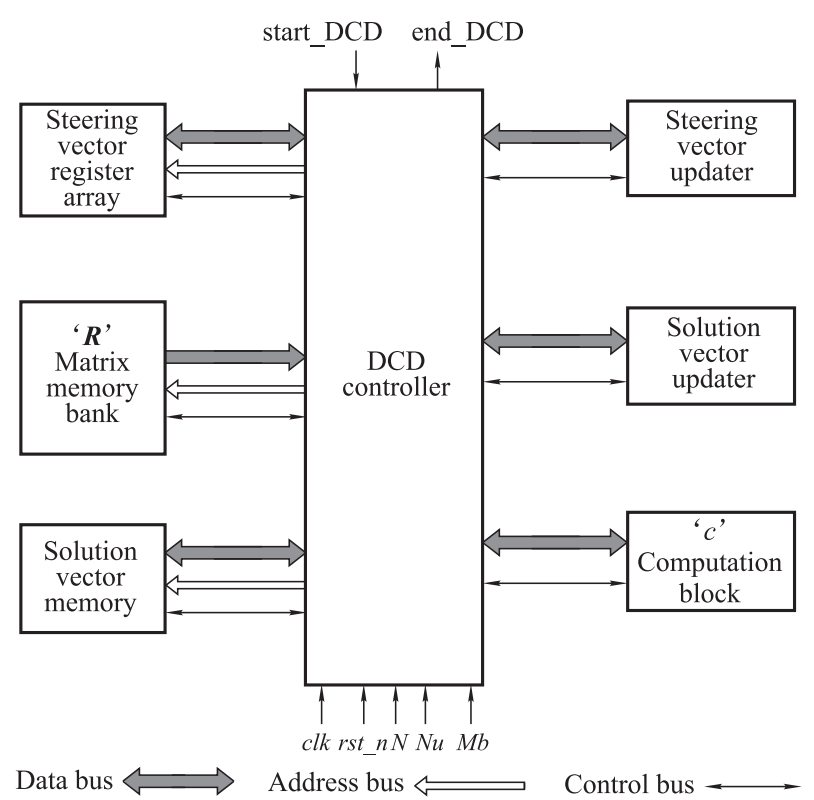

Fig. 3 Complex valued DCD division core architecture

The DCD controller proposed and implemented is shown in Table 3. The controller is implemented in three states, the initial state ( $\mathrm{S} 0$ ) and two operating states ( $\mathrm{S} 1$, $\mathrm{S} 2$ ). In the initial state, steering vector, correlation memory bank, number of bits to represent solution, various control flags, counters, etc., are initialized. Once start_DCD signal is encountered, the state moves to the first operating state S1. In S1, based on $s$ flag value real or imaginary value of steering vector from the current address pointer $n$ is assigned to a temporary register rtmp. Parallelly the constant $c$ value also will be updated based on the current correlation matrix diagonal value $R(n, n)$, rtmp and delm. In S1 state itself, based on $s$ flag, the next read address pointer will be incremented, and the associated read signals will be asserted. The state next moves to $\mathrm{S} 2$. The incremented read address and signals will be valid in S2 state and in S1 state the valid read data will always be generated. In S2 state, the solution vector $\boldsymbol{h}$ and steering vector $\boldsymbol{r}$ will be updated to the current write address pointer $n$, if constant value $c$ is less than zero. Also, the current next write address pointer $n$ will be updated. Based on the current control flags and count values, the next control flags and count values as well as the next state ( $\mathrm{S} 0$ or $\mathrm{S} 1$ ) are evaluated.

In this design, in state $\mathrm{S} 1$ the next read address counter $n$, read and write signals are asserted based on $c$ value and the next write address counter $n$ is not incremented. In state S2, these values will become the current value and hence updation will appear to the current write address counter $n$. The next write address counter $n$ will be incremented in $\mathrm{S} 2$ and will be assigned to the current state S1. Hence in S1 state, new values will be available from the current address $n$ and will be processed and updated in S2 state. Therefore, the number of clock cycles required per iteration is reduced to two.

The main arithmetic blocks in design are steering vector updater, solution vector updater and $c$ computation block. The steering vector $\boldsymbol{r}$ can be updated by (7) and the corresponding circuit design is depicted in Fig. 4.

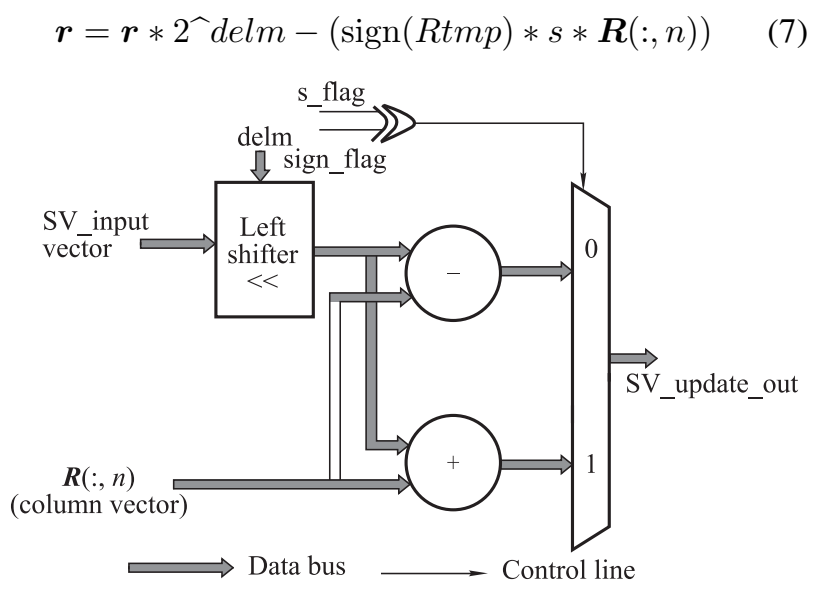

Fig. 4 Steering vector updater

The steering vector is left shifted delm times and added/subtracted parallelly with correlation matrix column vector $\boldsymbol{R}(:, n)$. The $s$ flag and $\operatorname{sign}(R t m p)$ flag are explored to generate the add/sub selection. The signed arithmetic is implemented by specifying reg signed keyword in Verilog register transfer level (RTL) description.

The solution vector is updated as per (8) and the similar circuit design and implementation to steering vector updater is adapted, except only one location of solution vector is updated per clock cycle.

$$
h(n)=h(n)+\operatorname{sign}(R t m p) * s * \alpha
$$


The constant $c$ computation logic is designed and implemented as depicted in Fig. 5 and the equation to implement the same is

$$
c=R(n, n) / 2-|R t m p| * 2^{\Upsilon} \operatorname{delm}
$$

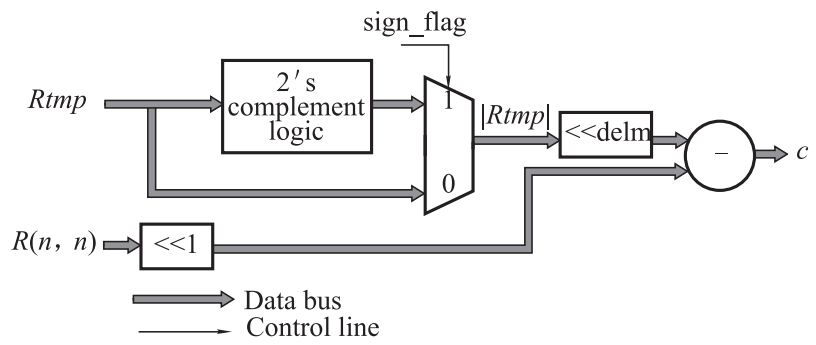

Fig. 5 c computation circuit

The arithmetic circuits are multiplication-free and involve only shift and add operations. They require only $O(N)$ shift and add/sub operations for steering vector updation and $O(1)$ shift and add/sub operation for solution vector and $c$ computation block. The combinational path delay involves a shifter, add/sub and a multiplexer delay; hence the design can be operated at a higher frequency.

A novel correlation memory bank structure which provides the diagonal element $R(n, n)$ and column vector $\boldsymbol{R}(:, n)$ in one clock is designed and implemented for DCD core. The structure is depicted in Fig. 6.

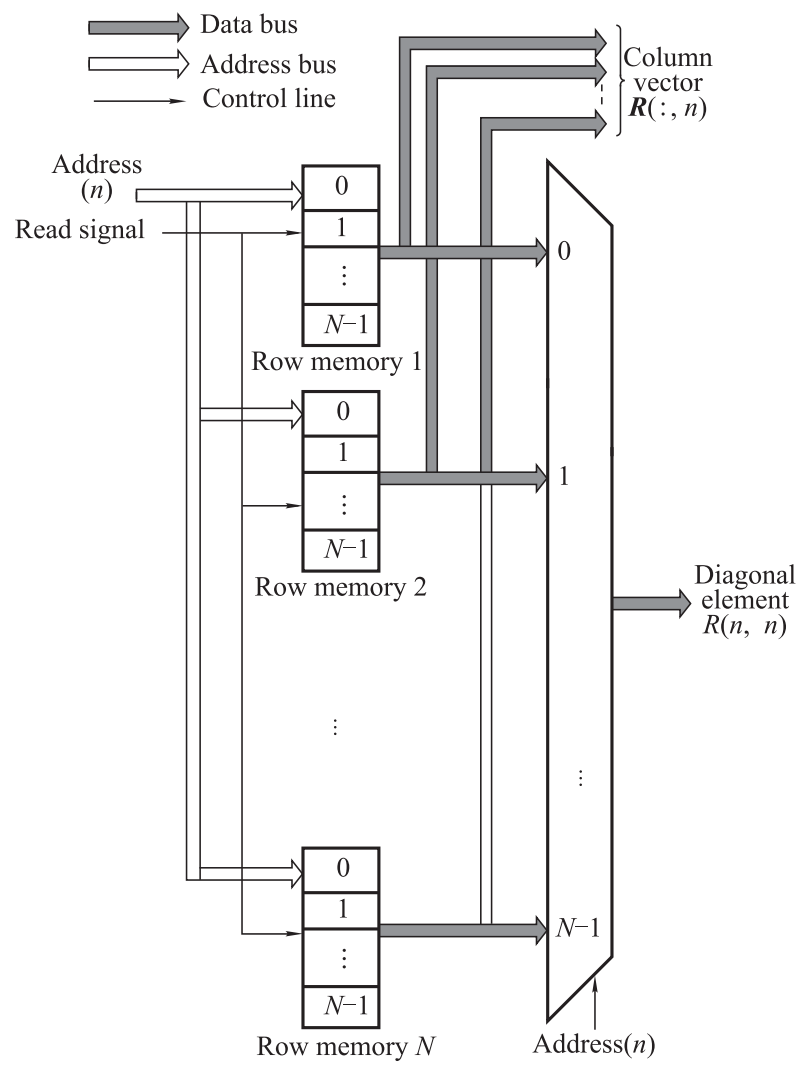

Fig. 6 Correlation matrix memory structure
In this structure, each row of the correlation matrix is stored in one block random access memory (RAM) of size $N \times 16$. Like this, $N$ block RAMs are utilized to form an $N \times N$ correlation matrix for both real and imaginary components. Based on the address $n$, the $n$th location content of all row memory as column vector, and specific row memory as diagonal element of the correlation matrix can be accessed. Hence in one clock cycle, the correlation vector, column vector and diagonal element can be accessed. We use true dual port RAM available in Xilinx block RAM generator for correlation matrix memory and solution vector memory design. The read and write operations are properly pipelined to achieve maximum throughput for DCD core.

\subsection{Complex vector MAC logic}

Fig. 7 depicts complex vector MAC logic. The multiplier unit contains up to 32 complex multiplier cores, and each core has four inputs and two outputs. As shown in Fig. 7, in each core, for getting real output, the adder adds the output from the first and second multipliers; for imaginary output, the subtractor subtracts the output from the third and fourth multipliers. All the 32 cores in complex multiplier perform the same operation. The 64 bit parallel adder adds the 32 real and 32 imaginary output form complex multiplier and generates single real and imaginary outputs.

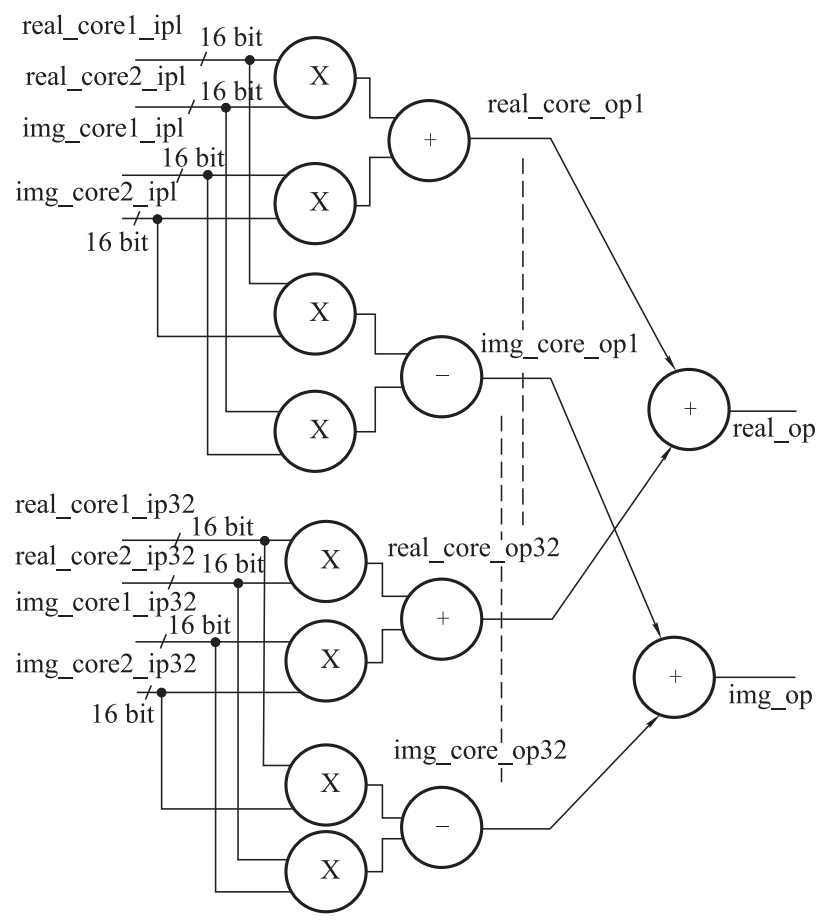

Fig. 7 Complex vector MAC logic architecture

\subsection{DCD divisor computation logic}

The divisor computation logic accepts the result of 
$\boldsymbol{S}(\boldsymbol{\Theta})^{\mathrm{H}} \boldsymbol{R}^{-1} \boldsymbol{S}(\boldsymbol{\Theta})$ as input and generates $\boldsymbol{R}^{\mathrm{T}} \boldsymbol{R}$ output. Fig. 8 illustrates the divisor computation logic. The real and imaginary part of $\boldsymbol{S}(\boldsymbol{\Theta})^{\mathrm{H}} \boldsymbol{R}^{-1} \boldsymbol{S}(\boldsymbol{\Theta})$ is formatted as $\left[\begin{array}{lll}d r & -d j ; d r & d j\end{array}\right]$ and fed to the divisor logic. It contains multipliers and adders to compute four $\mathrm{r}_{-}$transpose outputs.

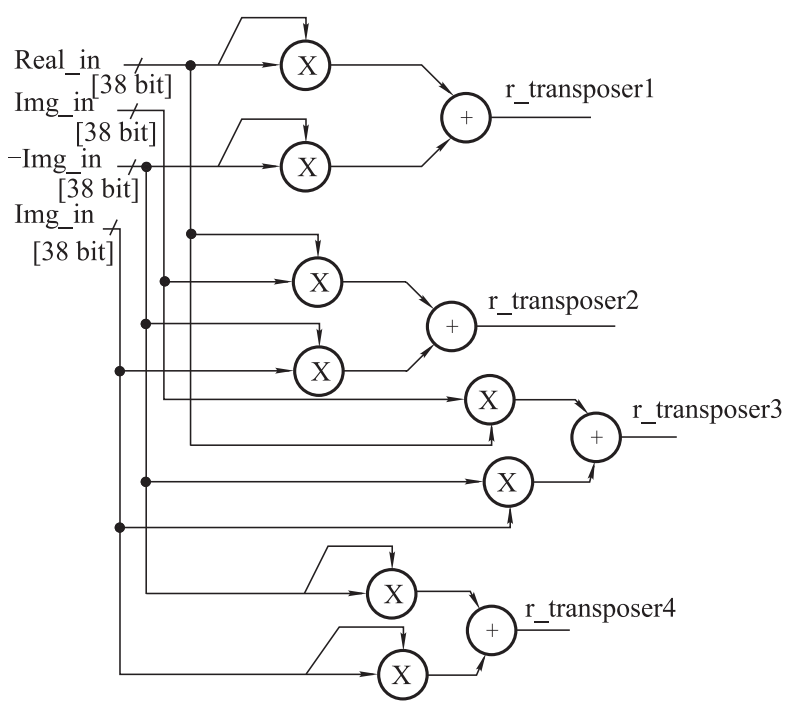

Fig. 8 DCD divisor computation logic architecture

\subsection{DCD dividend computation logic}

Fig. 9 shows the architecture of dividend computation logic. The output of complex vector MAC logic and each real and imaginary part of solution vector is fed to dividend computation logic. Fig. 9 illustrates only one core; like this up to 32 cores are employed to compute $q_{-}$core outputs in parallel.

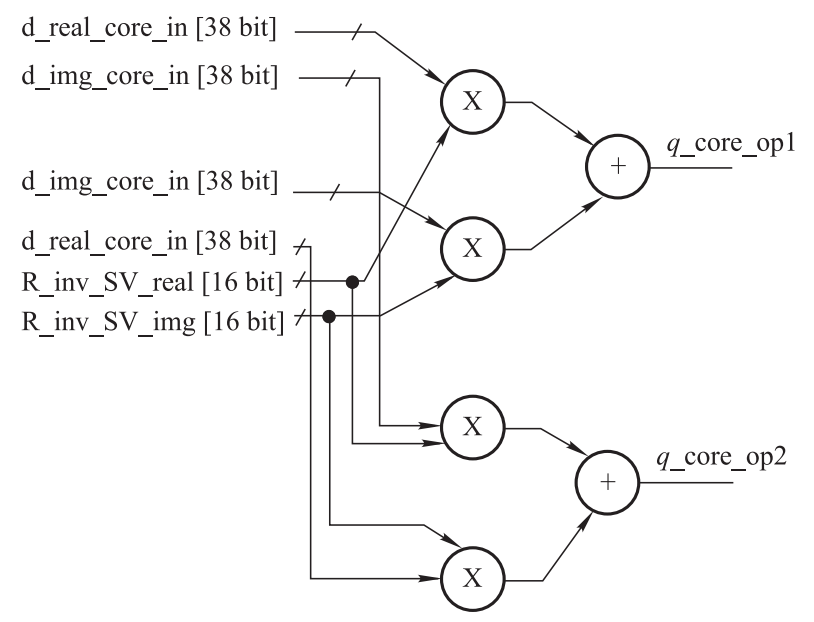

Fig. 9 DCD dividend computation logic

\subsection{Master controller}

The master controller generates the necessary control sig- nals to fetch steering vector register array and solution vector register array, and pipeline the operations among arithmetic units and DCD cores. It is implemented as five-state finite state machine (FSM); the states are the initial state, read state (read data from solution vector $(\mathrm{H})$ and steering vector (SV) register), start parallel DCD, h_read_enable state and adaptive weight register load state. Initially the controller is in S0 state; when start_div signal is asserted, the controller goes to read state S1. In S1, the controller generates parallel read signal and it reads data from $\mathrm{H}$ and SV register, then moves to S2 state. In S2 state, the start_DCD signal is asserted and it initiates the DCD computation. The controller waits in S2 state till the end_DCD signal is asserted, which indicates the DCD computation is over. Moment DCD computation is over, and the controller moves to S3 state then to S4 and further to S0 state. In S3 state, the controller generates write signal to adaptive weight register array and in S4 state, adaptive weight values will be written to register array.

\section{Results and discussions}

The proposed algorithms are first modeled in MATLAB programs, and bit accurate simulations are performed by generating uniform random values in range of $[-1,1]$ for steering vector and solution vector inputs and computing the adaptive weight vector. Maximum computational errors are recorded and verified within the theoretical bound. Further the proposed architecture is described in Verilog hardware description language (HDL), performs the simulations in Xilinx ISE 14.3 and generates the weight output. Fixed point implementation is followed, and steering vector, solution vector and weight outputs are represented in Q15 format. The random experiments are conducted; both MATLAB modeled and Xilinix ISE simulation results are compared and error is plotted (Fig. 10). The error between MATLAB and Verilog RTL simulations are in range of $35 \mathrm{~dB}$ to $-55 \mathrm{~dB}$ and well within the acceptable range as MVDR beamformer for medical imaging is concerned.

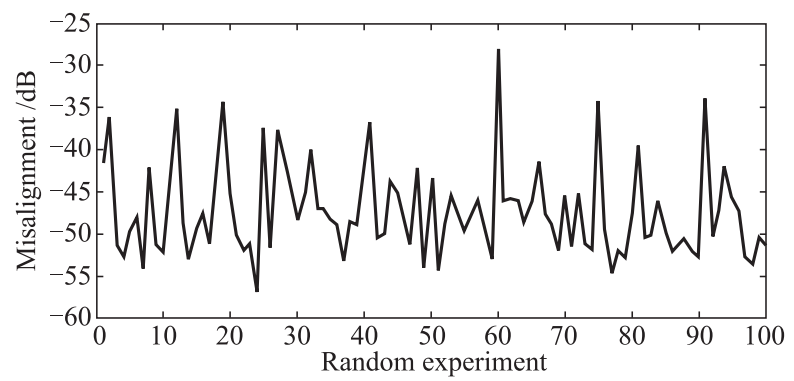

Fig. 10 Misalignment graph between MATLAB and Verilog RTL simulation results

The DCD core logic performs solution of a linear equa- 
tion of the form $\boldsymbol{A} \boldsymbol{x}=\boldsymbol{b}$, where $\boldsymbol{A}$ is a $2 \times 2$ matrix, $\boldsymbol{b}$ and $\boldsymbol{x}$ are $2 \times 1$ vectors. The proposed algorithm converges for $N=2$ and $M b$ (number of solution vector bits) $=16$ as depicted in Fig. 11. It converges approximately in 20 iterations. Compared to floating point counterpart MATLAB implementation, the error bound is approximately $-38 \mathrm{~dB}$. Since the modified FPGA DCD implementation takes only two clock cycles per iteration, the algorithm converges in 40 clock cycles. Hence by considering the overhead in computation of DCD division core inputs, the adaptive weight vector $(32 \times 1)$ will be computed in 43 clock cycles.

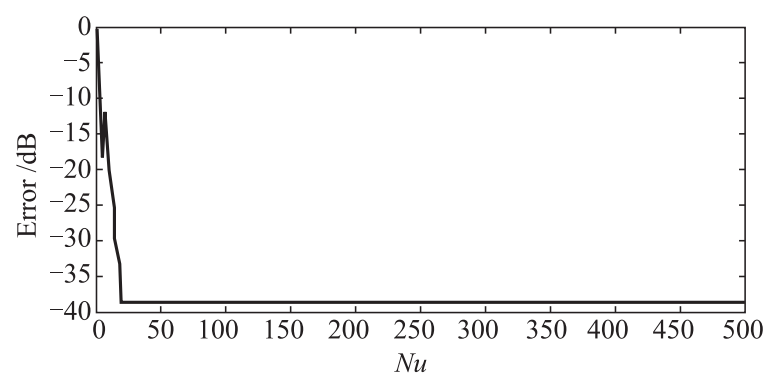

Fig. 11 Proposed DCD algorithm convergence graph for $N=2$, $M b=16$

The implemented architecture in Verilog RTL is ported on Xilinx Kintex-7 KC-705 FPGA evaluation platform and we could achieve frequency up to $213 \mathrm{MHz}$. Table 4 shows the resource utilization results in Kintex-7 FPGA platform. Table 4 shows that the architecture is highly efficient in area. Since the architecture is synthesized at a frequency of $213 \mathrm{MHz}$, one adaptive weight vector can be computed approximately in $200 \mathrm{~ns}$ and adaptive weight can be generated at a rate of $5 \mathrm{M}$ updates per second. As discussed in previous sections, typical weight updation rate requirement for an MVDR beamformer in medical ultrasound applications is approximately $52 \mathrm{~K}$ updates per second. As this division core could achieve up to 5M updates per second with fewer FPGA resources, this architecture can be easily adapted for implementing complex division in MVDR beamformer for medical ultrasound applications. Comparison of the proposed work with the existing studies is depicted in Table 5. CORDIC-based implementation [5] achieves a throughput of 113 MOPS, with a hardware cost of 4863 LUTs. Adapting the proposed architecture [5] in MVDR implementation for medical ultrasound applications is a nightmare as serial implementation causes huge controller overhead, and parallel implementation causes huge area and hence hardware realization of MVDR may not be feasible. Edman demonstrated an efficient throughput and area implementation of complex divider based on the Smith algorithm [6]. However, the Smith algorithm does not guarantee accurate results and hence this architecture cannot be adapted for medical imaging applications. Liu et al. showed a multiplicationand division-free complex division based on DCD iterations [7]. As DCD is multiplication- and division-free, it is area efficient, but as it is iterative, it takes longer converges time, hence reduction in throughput. The throughput achieved is 1.6 MOPS and adapting this architecture causes strict timing constrains on controller design.

Table 4 FPGA resource utilization

\begin{tabular}{ccc}
\hline FPGA resource & Total available & Used by logic \\
\hline Slice registers & 407600 & 8083 \\
Slice LUTs & 203800 & 26485 \\
Block RAMs & 445 & 96 \\
\hline
\end{tabular}

The proposed architecture addresses all those issues, and a modified complex cyclic valued DCD algorithm implementation on FPGA is devised to implement complex division in MVDR beamformer targeting medical ultrasound imaging applications. As compared to [7] we improve the throughput and resource utilization for DCD core. 32 DCD cores are parallel processed to generate up to $32 \times 1$ adaptive weight vectors, as shown in (1). As discussed previously, the modified DCD core achieves approximately 5 MOPS and by combining 32 cores in parallel, the whole division architecture can operate up to 160 MPOS to generate complex vector of $32 \times 1$. Also the total area including the complex vector MAC logic, DCD divisor and dividend computation logic is compromisable, enabling hardware implementation of MVDR beamformer to realize high resolution, real-time imaging applications. The existing works do not discuss power consumptions, and the proposed architecture consumes approximately $432 \mathrm{~mW}$ under peak operating conditions.

A broadband MVDR beamformer modeled in MATLAB [12] is used to validate the proposed architecture performance for hardware MVDR implementation. The ultrasound simulation program Field II is used to model the ultrasound echoes from simulation phantoms. Eight cyst phantoms positioned at $20 \mathrm{~mm}, 21 \mathrm{~mm}, 22 \mathrm{~mm}$, $50 \mathrm{~mm}, 60 \mathrm{~mm}, 70 \mathrm{~mm}, 80 \mathrm{~mm}$ and $90 \mathrm{~mm}$ are modeled to capture ultrasound echoes. The ultrasound pulse echoes for 32 sensors, at $4 \mathrm{MHz}$ center frequency and $100 \mathrm{MHz}$ sampling are generated for steering angles $\boldsymbol{S}(\boldsymbol{\Theta})-15^{\circ}$ to $+15^{\circ}$ at $1^{\circ}$ resolution. The weight vector values generated from FPGA simulation are used to generate the MVDR beamformer output for each steering angle, and the ultrasound image is generated. The same is compared with MATLAB simulation and shown in Fig. 12. It proves that the MVDR beamformer performs well with FPGA generated weights. 
Table 5 Comparison with the existing studies

\begin{tabular}{|c|c|c|c|c|c|c|c|c|}
\hline Scheme & $\begin{array}{c}\text { Latency/ } \\
\mathrm{ns}\end{array}$ & $\begin{array}{c}\text { Average } \\
\text { throughput/ } \\
\text { MOPS }\end{array}$ & Device & Area /LUT & Algorithm & Accuracy & $\begin{array}{l}\text { Number of } \\
\text { complex } \\
\text { divisions }\end{array}$ & $\begin{array}{c}\text { Precision/ } \\
\text { bit }\end{array}$ \\
\hline $\begin{array}{c}\text { The proposed } \\
\text { work }\end{array}$ & 201 & $\begin{array}{c}160 \\
\text { (32 div. units) } \\
5 \\
\text { (Single div. unit) }\end{array}$ & Xilinx Kintex-7 FPGA & $\begin{array}{c}26485 \\
\text { (32 div. units }+ \\
\text { control) } \\
621 \\
\text { (Single div. unit) }\end{array}$ & $\begin{array}{c}\text { DCD } \\
\text { (Modified complex } \\
\text { cyclic valued) }\end{array}$ & Very good & 32 & 16 \\
\hline $\begin{array}{l}\text { Wang } \\
\text { et al. [5] }\end{array}$ & 167 & 113 & Altera Straitix- FPGA & 4863 & CORDIC & Very good & 1 & 16 \\
\hline $\begin{array}{l}\text { Adman } \\
\text { et al. [6] }\end{array}$ & 80 & 16.67 & Xilinx Virtex-II FPGA & 117 (slices) & Smith & Poor & 1 & 16 \\
\hline $\begin{array}{c}\text { Liu } \\
\text { et al. [7] }\end{array}$ & 620 & 1.6 & Xilinx XC2VP30 FPGA & 527 (slices) & DCD & Very good & 1 & 16 \\
\hline
\end{tabular}
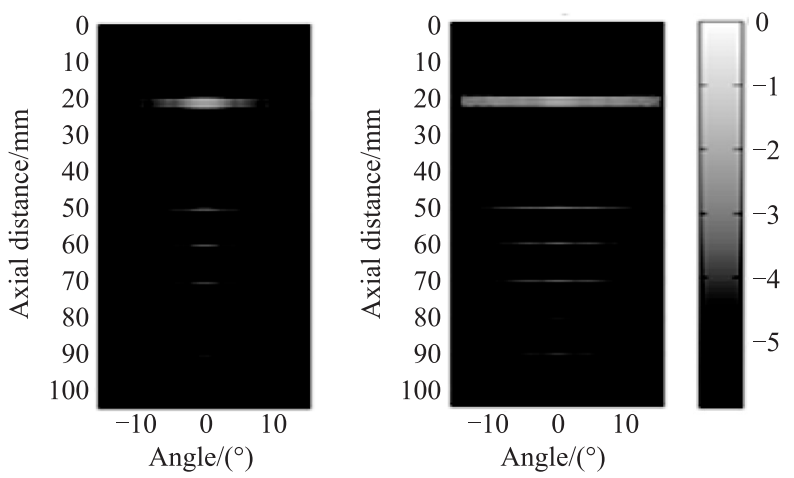

Fig. 12 Comparison of MVDR beamformer result with FPGA weight and MATLAB

The weight vectors generated by the proposed architecture are used to generate MVDR beamformer output for each steering angle and ultrasound image is formed. The generated image is compared with MATLAB simulated image and functionality is verified. The demonstrated architecture can be easily adapted to compute adaptive weight of MVDR beamformer for high resolution, sampling rate applications.

\section{Conclusions}

In this paper, a high-throughput and area-efficient fixed point complex divider architecture based on DCD iterations for complex division in adaptive weight computation stage of MVDR beamformer is demonstrated. The exiting complex valued cyclic DCD hardware implementation algorithm is modified for optimum performance. The implemented DCD core architecture for division can converge in 20 iterations and the whole complex division to generate adaptive weight vector can perform in 43 clock cycles. The 16 bit fixed point implementation on Xilinx Kintex7 XC320T FPGA shows approximately $3 \times$ advantage on throughput as compared to the existing DCD-based divider architecture without much cost in area. The proposed ar- chitecture is compared with other existing fixed point complex divider architectures and this paper concludes that the proposed architecture can be adapted to complex division in adaptive computation stage of broadband MVDR beamformer targeting medical ultrasound imaging applications.

The functionality of the architecture is validated by using the MATLAB simulation model developed for broadband MVDR beamformer using ultrasound simulation tool box Field II. The complex division for weight vector generation in adaptive weight computation stage is bypassed to the architecture and the adaptive weight vectors generated by the architecture are used to generate ultrasound scan lines and further the image. The generated image is compared with MATLAB counterpart and functionality is verified. Based on the results, it is concluded that the proposed architecture can be employed in adaptive weight computation stage of MVDR beamformer targeting medical ultrasound imaging applications. Also the proposed architecture can be extended to support higher number of channels with the cost of area.

\section{References}

[1] SYNNEVÅG J, AUSTENG A, HOLM S. Benefits of minimum-variance beamforming in medical ultrasound imaging. IEEE Trans. on Ultrasonics, Ferroelectrics, and Frequency Control, 2009, 56(9): $1868-1879$.

[2] PARK J, WI S M, LEE J S. Computationally efficient adaptive beamformer for ultrasound imaging based on QR decomposition. IEEE Trans. on Ultrasonics, Ferroelectrics, and Frequency Control, 2016, 63(2): 256-265.

[3] HOLFORTI K, GRAN F, JENSEN J A. Minimum variance beamforming for high frame-rate ultrasound imaging. Proc. of IEEE Ultrasonics Symposium, 2007: 1541 - 1544.

[4] HOLFORTI K, GRAN F, JENSEN J A. Broadband minimum variance beamforming for ultrasound imaging. IEEE Trans. on Ultrasonic, Ferroelectrics, and Frequency Control, 2009, 56(2): $314-325$.

[5] WANG D, REN P J, LIU L B. A high-throughput fixed point complex divider for FPGAs. IEICE Electronic Express, 2013, 10(4): $1-8$. 
[6] EDMAN F, ÖWALL V. Fixed-point implementation of a robust complex valued divider architecture. Proc. of the European Conference on Circuit Theory and Design, 2005: 143 146.

[7] LIU J, WEAVER B, ZAKHAROV Y. FPGA implementation of multiplication-free complex division. Electronics Letters, 2008, 44(2): 95 - 96.

[8] WRIGHTS J. Coordinate descent algorithms. Mathematical Programming: Series A and B, 2015, 151(1): 3-34.

[9] LIU J, ZAKHAROV Y, WEAVER B. Architecture and FPGA design of dichotomous coordinate descent algorithms. IEEE Trans. on Circuits Systems Part I: Regular Papers, 2009, 56(11): $2425-2438$.

[10] HEMA N, KIDAV J, LAKSHMI B. VLSI architecture for broadband MVDR beamformer. Indian Journal of Science and Technology, 2015, 8(19): 1-10.

[11] MA L, DICKSON K, MCALLISTER J. QR decomposition based matrix inversion for high performance embedded MIMO receivers. IEEE Trans. on Signal Processing, 2011, 59(4): $1858-1867$.

[12] UNNIKRISHNAN M, KIDAV J U. Broadband beamformer combined with DCDweighting. International Journal of Engineering Research in Electronic and Communication Engineering, 2015, 2(10): 7-11.

\section{Biographies}

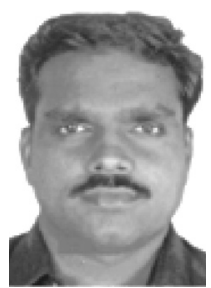

KIDAV Jayaraj U received his B.E. degree in electronics and communication engineering from Madurai Kamaraj University, India in 2000, and his M.E. degree in very-large-scale integrotion (VLSI) design from PSG College of Technology, Bharathiar University, India in 2002. Currently, he is pursuing his Ph.D. degree from Karunya Institute of Technology and Sciences, Coimbatore, India. His research focuses on high performance VLSI signal processing architecture development for adaptive beamformer in high sampling rate applications like medical ultrasound imaging, radar, etc. He joined as a scientist in Defense Research and Development Organization (DRDO), Ministry of Defense, Government of India in 2002, where he worked in signal processing systems development for defense applications. From
2008 to 2010, he worked as an R\&D engineer in Systems and Technology Group at IBM India Pvt. Ltd and currently he is working as a scientist at National Institute of Electronics and Information Technology (NIELIT) Calicut, Government of India.

E-mail: jayaraj@calicut.nielit.in

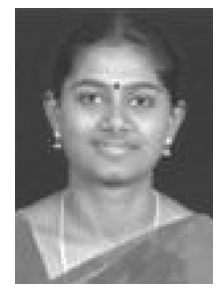

SIVA Mangai N M received her B.E. degree in electronics and communication engineering with distinction from the Madurai Kamaraj University, India in 2000, and her M.E. degree in VLSI design from PSG College of Technology, Bharathiar University, India in 2002. She completed her Ph.D. degree in information and communication engineering under Anna University, Chennai, India in 2011, focusing on power optimization and failure detection techniques for memory. She is a member of VLSI Society of India (VSI), International Association of Engineers (IAENG), International Association of Computer Science and Information Technology (IACSIT), International Congress for Global Science and Technology (ICGST) Academic Community and the Society of Digital Information and Wireless Communications (SDIWC), Institute of Doctors, Engineers and Scientists (IDES). She is currently working as an associate professor in electronics and communication engineering, Karunya University, Coimbatore, India.

E-mail: sivamangai@karunya.edu

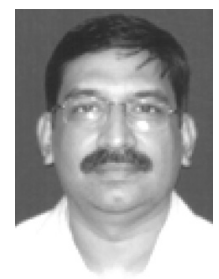

PERUMAL M Pillai received his Ph.D. degree in electronics (laser and EO engineering) from Dr. Babasaheb Ambedkar Marathwada University, Aurangabad India in 2006. He worked as a project/research associate in IIT Madras from 1989 to 1993, availed Swiss co-operation fellowship for eight months in 1996 and worked at Swiss Federal Institute of Technology, Lausanne. From 1993 to 1998 , he worked as a senior design engineer, from 1998 to 2006 as a principal design engineer and 2006 to 2010 as an additional director at CEDTI Aurangabd. From 2010 to 2013, he worked as a director in charge NIELIT Chennai. Currently he is working as an executive director of NIELIT Calicut. His research interests are in VLSI technologies, image processing techniques for medical imaging, fiber sensors \& distributed sensing, smart sensors, photonic \& fiber Bragg grating (FBG) devices.

E-mail: mppillai@calicut.nielit.in 